\title{
Herzfrequenz-Senker erstmals in den Leitlinien
}

\begin{abstract}
Die Leitlinien zur Herzinsuffizienz wurden in diesem Jahr beim Meeting der Experten in Belgrad aktualisiert und beim ESC-Kongress kommentiert.
\end{abstract}

Mit dem Frequenzsenker Ivabradin (Procoralan ${ }^{\circledast}$ ) hat es ein Neuling in den exklusiven Kreis der bei Herzinsuffizienz in den ESC-Leitlinien empfohlenen Pharmakotherapeutika geschafft. Springer Medizin hat bei Prof. Michael Böhm in dieser Angelegenheit nachgefragt:

CME: Herr Prof. Böhm, es gibt eine neue ESC-Leitlinie zum Thema Herzinsuffizienz. Was sind aus Ihrer Sicht die wesentlichen Neuerungen in der medikamentösen Therapie?

Böhm: Es gibt in den Leitlinien im Wesentlichen zwei Neuerungen. Das eine ist die Therapie mit Aldosteronantagonisten. Wir hatten zum Aldosteronantagonisten Eplerenon eine große Studie mit Patienten nach Herzinfarkt und eingeschränkter Pumpfunktion unter 30\% - im Mittel $26 \%$. Jetzt hat man bei Patienten mit stabiler Herzinsuffizienz in EMPHASIS-HF herausgefunden, dass die Patienten bei der Krankenhaussterblichkeit, den Hospitalisierungen und auch bei der Gesamtsterblichkeit profitieren.

Die Substanz wurde mit einer Klasse IA-Empfehlung in die Leitlinien aufgenommen, das heißt, die Substanz ist in jedem Fall indiziert. Nach der RALESStudie mit Spironolacton gibt es nun zwei Studien, die den günstigen Effekt der Mineralkortikoid-Rezeptorantagonisten auf das Überleben belegen. Die ESC hat sich entschlossen, eine Klassenempfehlung zu geben, also nicht eine einzelne Substanz empfohlen. Man sollte aber aus meiner Sicht die Substanz verwenden, bei der die Evidenz generiert wurde - und das ist das Eplerenon bei stabiler Herzinsuffizienz.

CME: Die zweite neu in die Leitlinie aufgenommene Substanz ist das Ivabradin.
Böhm: Die Substanz wurde in der SHIFT-Studie geprüft. Sie ist geeignet für Patienten mit einer relativ hohen Herzfrequenz, nämlich mehr als 70 Schläge pro Minute. Dann ist auch das Herzfrequenz-assoziierte Risiko erhöht. Es wird mehr Sauerstoff verbraucht und das Remodeling ist beschleunigt. SHIFT hat gezeigt: Wenn man nichts anderes macht als die Herzfrequenz zu reduzieren, kommt es zu einer 25-prozentigen Abnahme der Zahl der Klinikaufenthalte. Damit empfehlen die Leitlinien eine Ivabradin-Therapie von Patienten mit einer Herzfrequenz von mehr als 70 Schlägen pro Minute. Die Zulassung ist erfolgt aufgrund der Auswertung einer Subgruppe, die nicht präspezifiziert war, die eine Herzfrequenz von größer 75 Schlägen pro Minute hatte.

Die Botschaft ist: Patienten mit einer relativ hohen Herzfrequenz von 70-75 Schlägen pro Minute sollten Ivabradin erhalten.

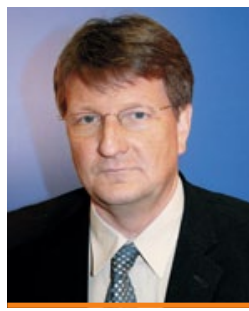

Prof. Dr. Michael Böhm

Universitätsklinikum des Saarlandes

Damit reduziert man etwa um ein Viertel die Zahl der Krankenhaus-Aufnahmen wegen einer Herzinsuffizienz. Die Studie lief unter einer optimalen Hintergrundtherapie mit Betablockern. Ivabradin ist also nicht einfach ein Ersatz für eine nicht ausgereizte Betablocker-Therapie. Betablocker, ACE-Hemmer, Aldosteron-Antagonisten sind die Basistherapie. Und dazu nun die Therapie mit Ivabradin.

(Rainer Klawki)

Cardio News 2012, 15(9):19; das komplette Interview auch unter: www.springermedizin.de/webtv

\section{Erfreulicher Trend}

\section{Weniger Tote bei Infarkt}

In den vergangenen 15-20 Jahren hat sich die Sterberate nach akutem ST-Strecken-Hebungs-Myokardinfarkt (STEMI) dramatisch verringert.

Auf diesen erfreulichen Trend sind Forscher in der FAST-MI-Studie gestoßen. Überraschend war aber auch, dass immer mehr Frauen schon in jüngeren Jahren einen STEMI erleiden. Lag die Rate für die 30-Tage-Mortalität im Jahr 1995 noch bei $13,7 \%$, betrug sie 2010 nur noch 4,4\%, berichtete Prof. Nicolas Danchin, eine relative Reduktion der Mortalität um 68\%. Bei Männern nahm sie von 9,8 auf 2,6\% ab, bei Frauen von 23,7 auf 9,8\%.

Eine Erklärung sind Verbesserungen der Therapie von Infarktpatienten. So erhöhte sich im Studienzeitraum der Anteil der STEMI-Patienten, bei denen eine frü- he Revaskularisation durch primäre Koronarintervention vorgenommen wurde, von 12 auf $61 \%$. Auch die Therapie mit Medikamenten wie ACE-Hemmer, Statine und Plättchenhemmer nahm deutlich zu.

Der Wermutstropfen: Der Anteil der weiblichen STEMI-Patienten unter 60 Jahren stieg von 11,8 auf $25,5 \%$, der Anteil der unter 50-Jährigen von 3,7 auf $11,1 \%$. Somit sei inzwischen ein Viertel aller Frauen, die von STEMI betroffen sind, jünger als 60 Jahre. Erklären lässt sich der ungünstige Trend vor allem durch die stetig wachsende Zahl von jungen Raucherinnen. Im Jahr 1995 waren nur 37\% aller jüngeren Frauen mit Herzinfarkt Raucherinnen, im Jahr 2012 dagegen $73 \%$, berichtete Danchin.

(ob) 